\title{
Current and Emerging Imaging Techniques in Patients with Genetic Aortic Syndromes
}

\section{Aktuelle Bildgebungs-Strategien bei genetisch bedingten Erkrankungen der Aorta}

Authors

Julius Matthias Weinrich¹, Alexander Lenz ${ }^{1}$, Evaldas Girdauskas², Gerhard Adam ${ }^{1}$, Yskert von Kodolitsch ${ }^{3}$, Peter Bannas ${ }^{1}$

Affiliations

1 Department of Diagnostic and Interventional Radiology and Nuclear Medicine, University Medical Center Hamburg-Eppendorf, Hamburg, Germany

2 Department of Cardiovascular Surgery, University Heart Center Hamburg, Hamburg, Germany

3 Department of Cardiology, University Heart Center Hamburg, Hamburg, Germany

Key words

genetic aortic syndrome, Loeys-Dietz-syndrome, Ehlers-Danlos-syndrome, Marfan-syndrome

received 24.12.2018

accepted 24.04.2019

Bibliography

DOI https://doi.org/10.1055/a-0914-3321

Published online: 6.6.2019

Fortschr Röntgenstr 2020; 192: 50-58

(c) Georg Thieme Verlag KG, Stuttgart · New York

ISSN 1438-9029

Correspondence

Dr. Julius Matthias Weinrich

Department of Diagnostic and Interventional Radiology

and Nuclear Medicine, University Medical Center Hamburg-

Eppendorf, Hamburg, Germany, Martinistraße 52,

20246 Hamburg, Germany

Tel.: ++ 49/40/741054010

J.weinrich@uke.de

\section{ABSTRACT}

Background Patients with genetic aortic syndromes such as Marfan or Loeys-Dietz syndrome have a decreased life expectancy due to the risk of aortic dissection and rupture. Imaging plays an important role in the acute setting but also in the initial diagnosis and image-based monitoring. In this article, we provide an overview of the most common genetic aortic syndromes and recommended imaging strategies. Furthermore, we highlight modern imaging methods allowing for the quantification of hemodynamic changes in aortic disease. Method This is a narrative review article on genetic aortic syndromes and recommended imaging strategies, where we take into account expert opinions and standard-of-care practices from our own center.

Results and Conclusion Radiological imaging plays a key role in the initial diagnosis and surveillance of patients with genetic aortic syndromes. Radiologists contribute significantly to the multi-disciplinary setting of genetic aortic syndromes with knowledge of special features and recommended imaging methods. Accurate measurement of the aorta is crucial, particularly in terms of diameter-based surgical treatment algorithms. Modern imaging methods like 4D-flow MRI and pulse wave velocity have a potential to further improve individualized risk stratification in patients with genetic aortic syndromes.

Key points:

- The risk for cardiovascular complications such as acute aortic syndrome is increased in patients with genetic aortic syndromes.

- Recommended time intervals between image-based monitoring depend on the underlying aortic disease.

- CT-angiography should be used only in the acute setting.

- Non-contrast MR-angiography is adequate for screening and image-based monitoring of patients with genetic aortic syndromes.

\section{Citation Format}

- Weinrich JM, Lenz A, Girdauskas E et al. Current and Emerging Imaging Techniques in Patients with Genetic Aortic Syndromes. Fortschr Röntgenstr 2020; 192: 50-58

\section{ZUSAMMENFASSUNG}

Hintergrund Genetische Aortenerkrankungen wie das Marfan- oder Loeys-Dietz-Syndrom umfassen ein relativ kleines, jedoch wichtiges kardiovaskuläres Patientenkollektiv. Die betroffenen Patienten unterscheiden sich durch diverse Phänotypen, haben jedoch alle bereits in jungem Alter ein sehr hohes Risiko für ein akutes Aortensyndrom. Sowohl in der Akutsituation als auch im Rahmen der Vorsorge ist die Schnittbildgebung unverzichtbar. In diesem Übersichtsartikel stellen wir die häufigsten genetischen Aortenerkrankungen und die für sie empfohlene Bildgebung vor. Darüber hinaus geben wir einen Ausblick auf moderne Methoden zur Erfassung der Hämodynamik bei Aortenerkrankungen. 
Methode Diese Übersichtsarbeit basiert auf der Kombination publizierter Expertenmeinungen sowie den klinischen Standards unseres auf Aortenerkrankungen spezialisierten Zentrums.

Ergebnisse und Schlussfolgerungen Die radiologische Bildgebung ist zentraler Bestandteil der initialen Diagnosestellung und Verlaufskontrolle genetischer Aortenerkrankungen und unterscheidet sich in Abhängigkeit der Grunderkrankung. Durch Kenntnis der Besonderheiten genetischer Aortenerkrankungen und der empfohlenen Bildgebungstech- niken trägt der Radiologe wesentlich zur optimalen Patientenbetreuung bei. Die genaue Vermessung der Aortendiameter beeinflusst maßgeblich den interdisziplinären Beschluss eines prophylaktischen chirurgischen Aortenersatzes. Moderne Bildgebungstechniken wie die 4D-Fluss-MRT und die Pulswellengeschwindigkeit haben das Potenzial, die individualisierte Risikostratifizierung bei Patienten mit genetischen Aortenerkrankungen zu verbessern.

\section{Background}

With a prevalence of $1.5-17.2 / 100000$, Marfan syndrome is the most common genetic aortic syndrome [1, 2]. There are also a number of other genetic aortic syndromes including Ehlers-Danlos syndrome and Loeys-Dietz syndrome [3]. Even in the case of non-syndromic acute aortic syndrome, approximately $20 \%$ of patients have a genetic predisposition [4]. A common feature of all genetic aortic syndromes is an increased risk for aneurysms and associated life-threatening aortic dissection [3]. Therefore, radiologists must be familiar with the most common genetic aortic syndromes and the recommended imaging for each. Knowledge of the correct imaging method and exact measurement of the aortic diameter are relevant for diagnosis, individual risk stratification, the timing of prophylactic aortic replacement surgery, and for the planning of complex interventions [5].

Based on published expert opinions and the clinical standards of a center specialized in aortic syndromes, the following presents the most common genetic aortic syndromes and the recommended imaging methods. Moreover, we provide an overview of modern methods such as $4 \mathrm{D}$ flow MRI and MRI-based measurement of the pulse wave velocity in aortic diseases.

\section{Imaging methods}

\section{Transthoracic echocardiography (TTE)}

TTE is a cost-effective and widely available imaging method allowing visualization of the aortic root ( $\vee$ Fig. 1 ) and also portions of the ascending aorta and the aortic arch [6]. In addition to aortic diameters, TTE can also be used to assess valve and ventricular function.

A majority of the studies regarding standard aortic root diameter values are based on echocardiographic values [7]. Because of decades of clinical experience, TTE is an integral part of the initial examination in the case of suspicion of a genetic aortic syndrome. However, genetic aortic syndromes are often associated with changes in habitus [8]. Particularly in Marfan syndrome, thoracic deformities such as a Pectus excavatum are often seen thus complicating visualization of the aortic root.

In summary TTE continues to be important for initial diagnosis as well as for follow-up due to its broad availability and the extensive clinical experience.

\section{Computed tomography angiography (CTA)}

CTA is widely available and can visualize the entire aorta quickly and with high resolution [7]. Post-procedural multiplanar reconstruction represents a further advantage. As a result, arbitrary image planes can be generated allowing the orthogonal and thus exact measurement of the aortic diameter. CTA is the reference standard for ruling out dissection in the case of acute aortic syndrome ( $\bullet$ Fig. 1 ) [6].

Exposure to ionizing radiation and the administration of intravenous iodine-containing contrast agent are limitations of CTA [5]. Since young patients with genetic aortic syndrome often need to be examined via cross-sectional imaging repeatedly over the course of their lives, CTA should only be used in acute situations or in preoperative or preinterventional treatment planning [3]. CTA should not be used in preventive care.

\section{Magnetic resonance angiography (MRA)}

MRA allows multiplanar cross-sectional imaging of the entire aorta without the use of ionizing radiation [9], precise measurement of the aortic diameter [10] and diagnosis of aortic dissections [6] with high diagnostic accuracy. It can also be used for quantification of possible insufficiencies or stenoses of the aortic valve. The disadvantage of MRA in comparison to CTA is the longer examination time.

MRA can be performed both with and without administration of intravenous contrast media. The advantages of contrast-enhanced MRA include short acquisition times, high spatial resolution, and the ability to assess the perfusion of a false lumen in the case of a dissection [11]. The acquisition of $3 \mathrm{D}$ sequences with isotropic datasets allows multiplanar reconstructions (MPRs), which can be used for optimal measurement of the aortic diameter.

Multiple studies have been able to show that ECG-triggered non-contrast MRA, e. g. using steady-state free precession (SSFP) sequences, is equal to contrast-enhanced MRA for measuring the aortic diameter in preventive care $[9,12,13]$. Postoperative follow-up after aortic replacement surgery can also be performed using non-contrast MRA [14]. Therefore, we recommend non-contrast ECG-triggered MRA for the annual follow-up of asymptomatic patients with a genetic aortic syndrome. Using this method, the entire aorta can be visualized and measured without the risks associated with ionizing radiation or intravenous contrast agents. 

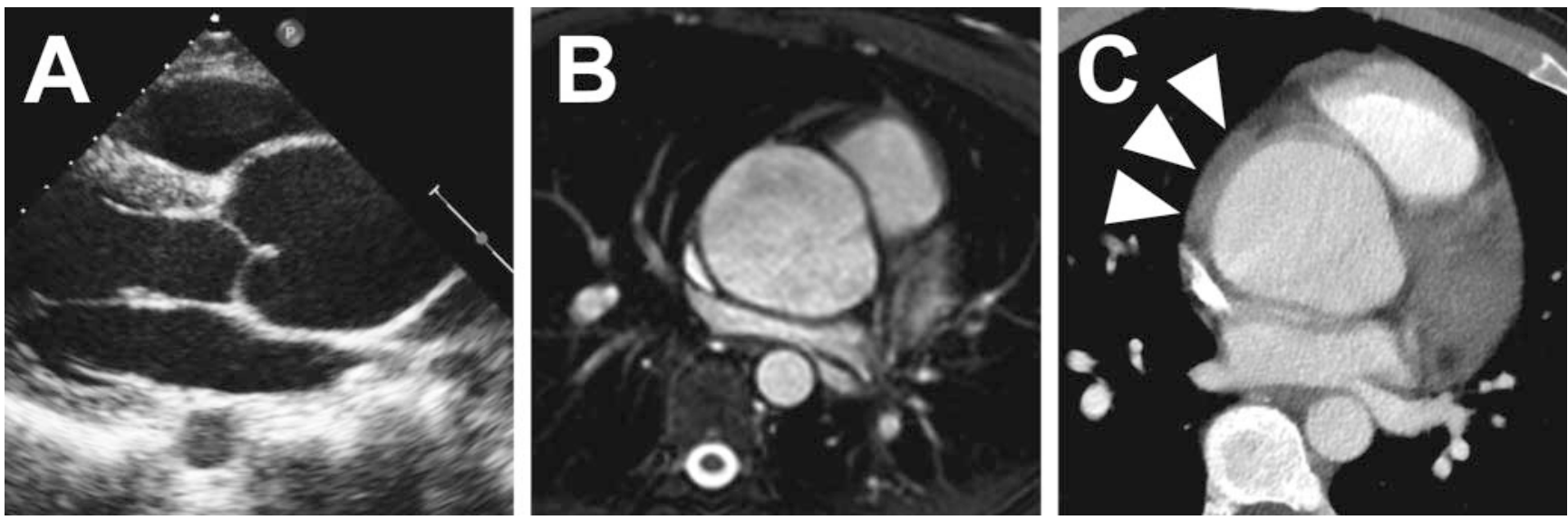

- Fig. 1 21-year-old Loeys-Dietz patient with aneurysm of the aortic bulb on echocardiography $\mathbf{A}$ and non-contrast MRA B in screening and with intramural hematoma on CTA $\mathbf{C}$ in acute aortic syndrome. In this patient the indication for surgical aortic root replacement was determined during screening based on the aneurysm of the aortic bulb $(5.9 \mathrm{~cm})$ detected on echocardiography and MRA. Two weeks prior to the planned surgery, the patient presented with an acute aortic syndrome at the emergency room. Emergency contrast-enhanced CTA showed an intramural hematoma (IMH) (C, arrowheads). The patient was able to be saved by emergency surgery.

\section{D flow MRI and pulse wave velocity}

To date, the absolute aortic diameters and the annual growth rate of an aortic aneurysm are considered the most important predictors for life-threatening aortic dissection [6]. However, dissections also occur in patients with genetic aortic syndromes with normal diameter [15]. This highlights the necessity to identify additional predictors for dissection and the importance of investigating new hemodynamic parameters and their effect on the vascular wall.

One of these parameters is the eccentricity of the blood flow in the vessel, which can be quantified by $2 \mathrm{D}$ phase contrast (PC) MRI. It was recently able to be shown in patients with bicuspid aortic valve disease that blood flow eccentricity is predictive for an increase in the diameter of the ascending aorta [16].

4D PC MRI (4 D flow MRI) allows co-registration of morphological images and velocity data $[17,18]$ and thus the visualization and quantification of physiological and pathological blood flow profiles [19-21]. In large vessels like the aorta, 4D flow MRI allows the visualization and quantification of complex changes in hemodynamics, e. g. vortices and helices ( $\triangleright$ Fig. 2) [17, 22]. Moreover, the wall shear stress can be derived from 4D flow MRI as a measure for the forces acting on the aortic wall [23]. A correlation between hemodynamic changes and the morphology of the aorta can be seen in patients with a bicuspid aortic valve as well as with Marfan syndrome [24-26]. These MRI-based studies support the theory that pathological blood flow profiles (flow eccentricity, helix, vortex, wall shear stress) contribute to the formation of aneurysms.

Changes in global or regional vascular stiffness were also described as a risk factor for the progression of aortic dilatation in patients with Marfan syndrome [27]. The vascular stiffness of the aorta can be derived indirectly from the pulse wave velocity (PWV). The PWV is elevated in the case of a stiff vascular wall and can be determined noninvasively via PC MRI ( $\bullet$ Fig. 2) [28]. Compared to healthy subjects, patients with Marfan syndrome have an elevated pulse wave velocity [29]. It was able to be shown that pulse wave velocity can predict aortic dilatation [30].
These initial results regarding 4D flow MRI and pulse wave velocity indicate their potential for the future assessment of aortic diseases. Large prospective studies with clinically relevant end points are necessary to confirm the diagnostic and prognostic value of these MRI techniques.

\section{Measuring aortic diameter}

Precise measurement of the aortic diameter is essential in patients with genetic aortic syndromes [5, 31]. Dilatation of the aortic bulb to $5 \mathrm{~cm}$ is an indication for prophylactic aortic replacement in Marfan patients and in patients with a bicuspid aortic valve and additional risk factors (i. e., positive family history, hypertension, fast growth: $>3 \mathrm{~mm} /$ year) [6]. Annual growth of $>3 \mathrm{~mm}$ is also a risk factor for aortic dissection in Marfan patients [6]. Therefore, standardized measurement methods are necessary: The aortic diameter must be measured in a standardized manner at certain aortic segments ( $\bullet$ Fig. 3 ). The aortic diameter must be measured orthogonally to the anatomical axis of the aorta. Therefore, measurement cannot be performed on axial CTA or MRA source images [32]. Mendoza et al. were able to show on the basis of CTA data that, compared to orthogonal diameters, diameters measured on a strictly axial basis double the diameter-based indication for surgery [33].

In patients with genetic aortic syndromes, standardized measurements are very important due to the need for repeated followup examinations. The aortic diameter should be measured both in the last (most recent) and the first (oldest) baseline examination, ideally performed by the same radiologist [34]. In our experience slow increases in diameter in the millimeter range can be reliably identified and gradual growth can be evaluated only in this way.

The measured aortic diameters are converted to a Z-score particularly in pediatrics. Z-scores describe in standardized form the extent to which a measured value deviates from the expected mean of a comparison group. The Z-score is adapted to patient age and body surface [35]. 

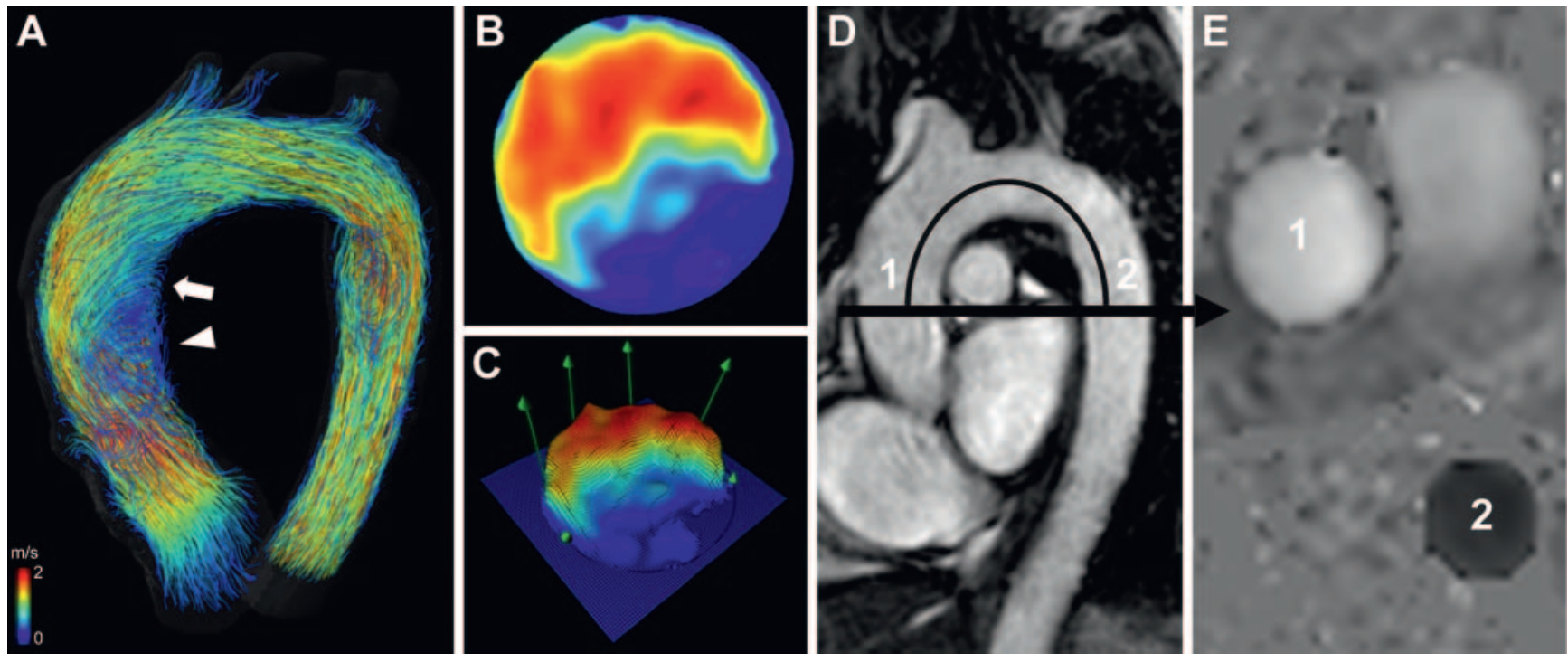

- Fig. 2 4D phase contrast (4D flow) MRI in a 24-year-old patient with bicuspid aortic valve disease A-C and 2D phase contrast MRI for determining the pulse wave velocity in a 31-year-old patient with Marfan syndrome D, E. 4D flow MRI-based parasagittal visualization of pathological helical (arrow) and vortical (arrowheads) flow patterns in the ascending aorta due to the asymmetrical and stenotic bicuspid aortic valve. Orthogonal cross-section of the aorta with $\mathbf{B}$ velocity-encoded visualization of the eccentric and accelerated blood flow along the vascular wall and $\mathbf{C}$ vectorbased visualization of the wall shear stress in the ascending aorta (length of the vectors = degree of wall shear stress). D Parasagittal SSFP-MRA with measurement planes orthogonal to the ascending (1) and descending (2) aorta with the distance between them being measured. E 2D phase contrast MRI is used to determine the time difference of the pulse waves between the ascending (1) and descending (2) aorta. The pulse wave velocity is calculated from the quotient of the distance between the two measurement points and the time difference of the blood flow.

In summary, the aortic diameter must be determined in a standardized manner. Therefore, we recommend creating process instructions and a note in the radiology report as to the slice plane that was used for the aortic diameter measurement.

\section{Genetic aortic syndromes: Etiology, prevalence and special features of imaging}

\section{Marfan syndrome}

Marfan syndrome is a systemic autosomal dominant genetic connective tissue disorder caused by a mutation of the fibrillin 1 gene (FBN1). Marfan syndrome occurs with a rate of 1.5-17.2/ 100000 [1, 2]. Marfan syndrome is diagnosed based not only on a gene mutation but also on additional clinical criteria as defined in detail in the 2010 revised Ghent Nosology [1]. Aortic dilatation is a decisive criterion here.

In Marfan syndrome, the risk of an early death due to aortic rupture or dissection is significantly increased without regular image-based follow-up [6]. Dissection is typically, but not necessarily, preceded by aneurysmatic dilatation of the aortic root ( $\triangleright$ Fig. 4A, B) [36]. However, aneurysms and dissections of the distal aorta can also occur in patients following proximal aortic replacement [37]. Thus, imaging of the entire aorta is not only decisive for the initial diagnosis and follow-up ( $\mathbf{F i g . 5 A}, \mathbf{B}$ ) but also for monitoring in the case of aortic replacement [38]. Accordingly, patients with Marfan syndrome require imaging of the aorta throughout their lives.
The interdisciplinary American guidelines recommend performing TTE for the initial diagnosis and at 6-month intervals to assess the aortic root and the proximal ascending aorta [5]. In the case of a constant diameter, annual imaging is recommended. If an absolute diameter of $4.5 \mathrm{~cm}$ is exceeded or in the case of significant growth more frequent imaging is recommended [5].

\section{Loeys-Dietz syndrome}

Loeys-Dietz syndrome is an autosomal dominant genetic connective tissue disorder with a prevalence of 1/100 000 first described in 2005 [39, 40]. It has similarities with Marfan syndrome but has major differences regarding the phenotypic presentation as well as the prognosis [41]. The known subtypes (I-IV) are based on gene mutations of transforming growth factors $\beta$ receptors 1 and 2 (TGFBR1 and TGFBR2) and the decapentaplegic homolog 3 proteins (SMAD3 and TGFB2) occurring in the same signaling pathway. The prognosis for types I and II is most unfavorable since aortic ruptures can occur in these patients even in the case of normal vessel diameters [39]. Type I of the Loeys-Dietz syndrome is characterized on the vascular level primarily by aneurysms of the large vessels and pronounced tortuosity of the smaller vessels ( $\triangleright$ Fig. 6). In contrast, type II can resemble vascular Ehlers-Danlos syndrome and has a higher peri/postoperative complication rate than the other subtypes (Loeys-Dietz syndrome general: $1.7 \%$; type II: $4.8 \%$ ) [42]. The diagnosis must be definitively confirmed since the indication for surgery is determined more liberally for Loeys-Dietz syndrome than for vascular Ehlers-Danlos syndrome due to the lower incidence of peri/postoperative complications (45\%) [42, 43]. In contrast to Marfan syndrome, aneurysms are frequently abdominal or intracranial in Loeys-Dietz syndrome 


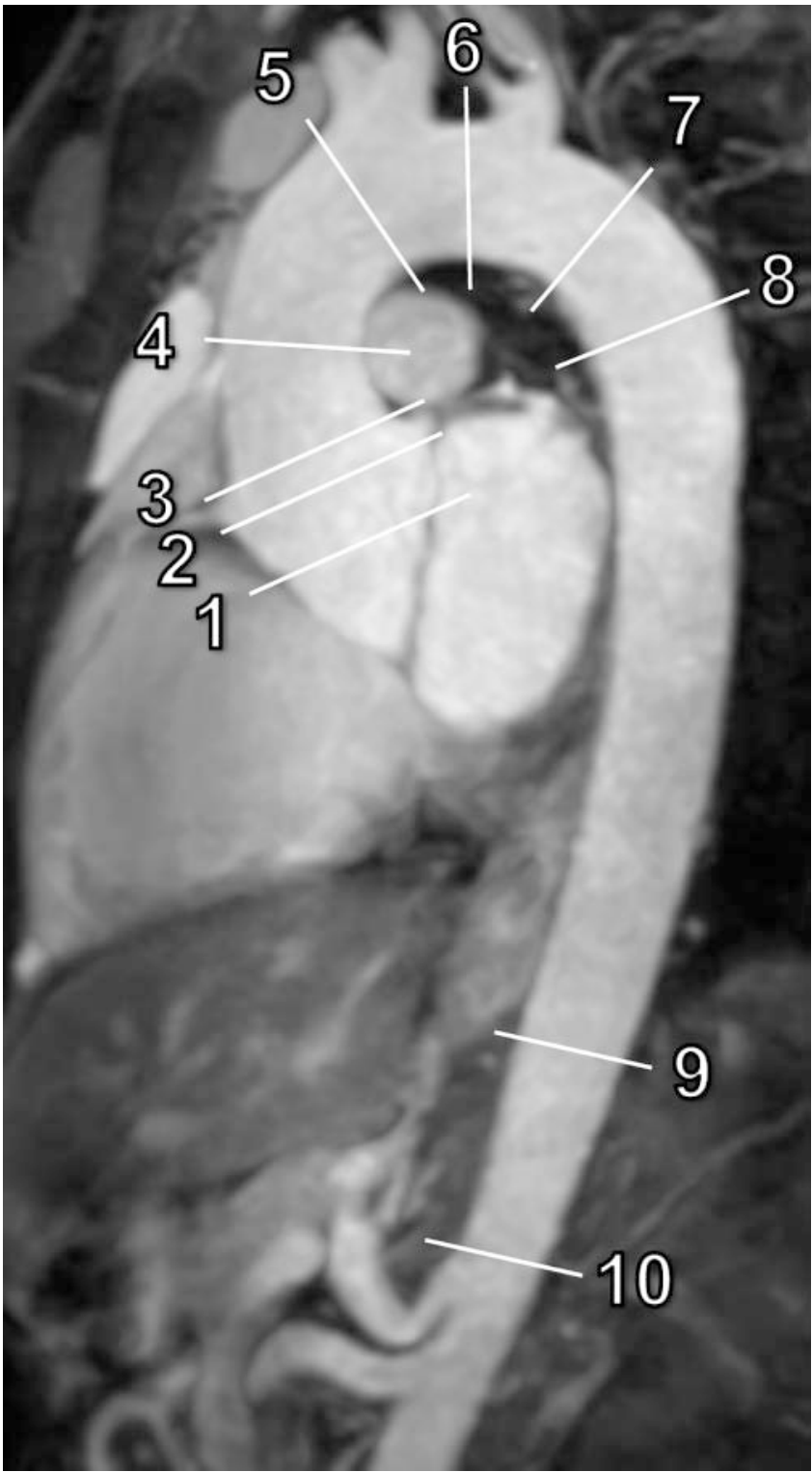

- Fig. 3 Parasagittal reconstruction of non-contrast 3D-MRA with illustration of recommended aortic measurement planes. The following aortic segments should be measured in a standardized manner: 1: Aortic annulus; 2: Aortic bulb/sinus valsalva; 3: Sinotubular junction; 4: Ascending aorta on the level of the pulmonary trunk; 5: Proximal aortic arch at the origin of the brachiocephalic trunk; 6: Middle aortic arch between the left common carotid artery and left subclavian artery; 7: Proximal descending aorta $2 \mathrm{~cm}$ distal to the left subclavian artery; 8: Descending aorta on the level of the pulmonary trunk; 9: Aorta on the level of the diaphragmatic opening; 10: Aorta above the celiac trunk.

and the disease is more aggressive [15]. In addition to aneurysms, Loeys-Dietz syndrome is associated with a patent ductus arteriosus, atrial septal defects, and tortuosity of smaller arteries [3].

The interdisciplinary American guidelines recommend closer image-based monitoring ranging from the intracranial to the pelvic vascular system for Loeys-Dietz syndrome in comparison to Marfan syndrome [5]. CTA/MRA of the entire aorta should be performed at the time of diagnosis and after 6 months. Whole-
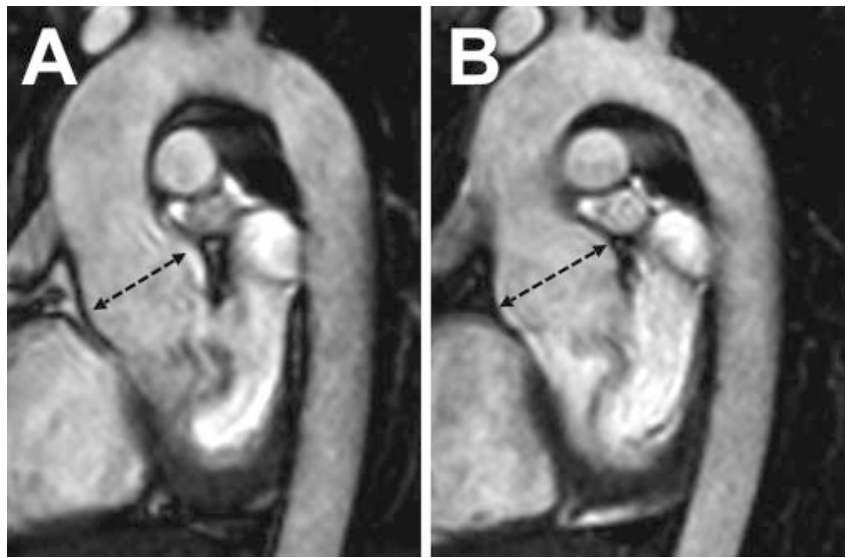

- Fig. 4 Sagittal non-contrast SSFP-MRA of the thoracic aorta of a 35-year-old patient with Marfan syndrome with progressive dilatation of the aortic bulb (A: prior image; B: current image). The aortic bulb increased in diameter from $3.7 \mathrm{~cm}$ A to $4.4 \mathrm{~cm}$ B within three years.
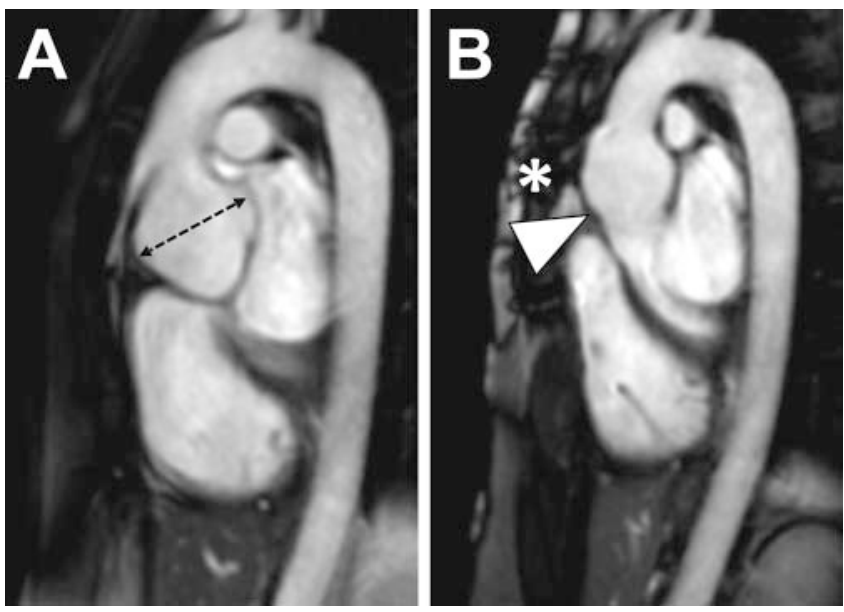

- Fig. 5 33-year-old Marfan patient before $\mathbf{A}$ and after $\mathbf{B}$ aortic root replacement on sagittal non-contrast SSFP-MRA of the thoracic aorta. A Due to the $5.1 \mathrm{~cm}$ aneurysm of the aortic bulb (dashed line) detected during screening, elective surgery was indicated. A valvesparing aortic root replacement according to David in combination with a valsalva prosthesis was performed. B The aorta can be effectively evaluated postoperatively on non-contrast SSFP-MRA despite the presence of sternal cerclages causing susceptibility artifacts (B, star).

body MRA is recommended for the further annual follow-up examinations [5]. The frequency of imaging increases as a function of the findings and regular intervals of at least two years are recommended.

\section{Ehlers-Danlos syndrome}

Ehlers-Danlos syndrome includes a genetically and clinically heterogeneous group of connective tissue diseases with 13 subtypes and a prevalence of $4-10 / 100000[44,45]$. Due to rupture of vessels or internal hollow organs, particularly the colon and uterus, Ehlers-Danlos syndrome has a high mortality rate with a 


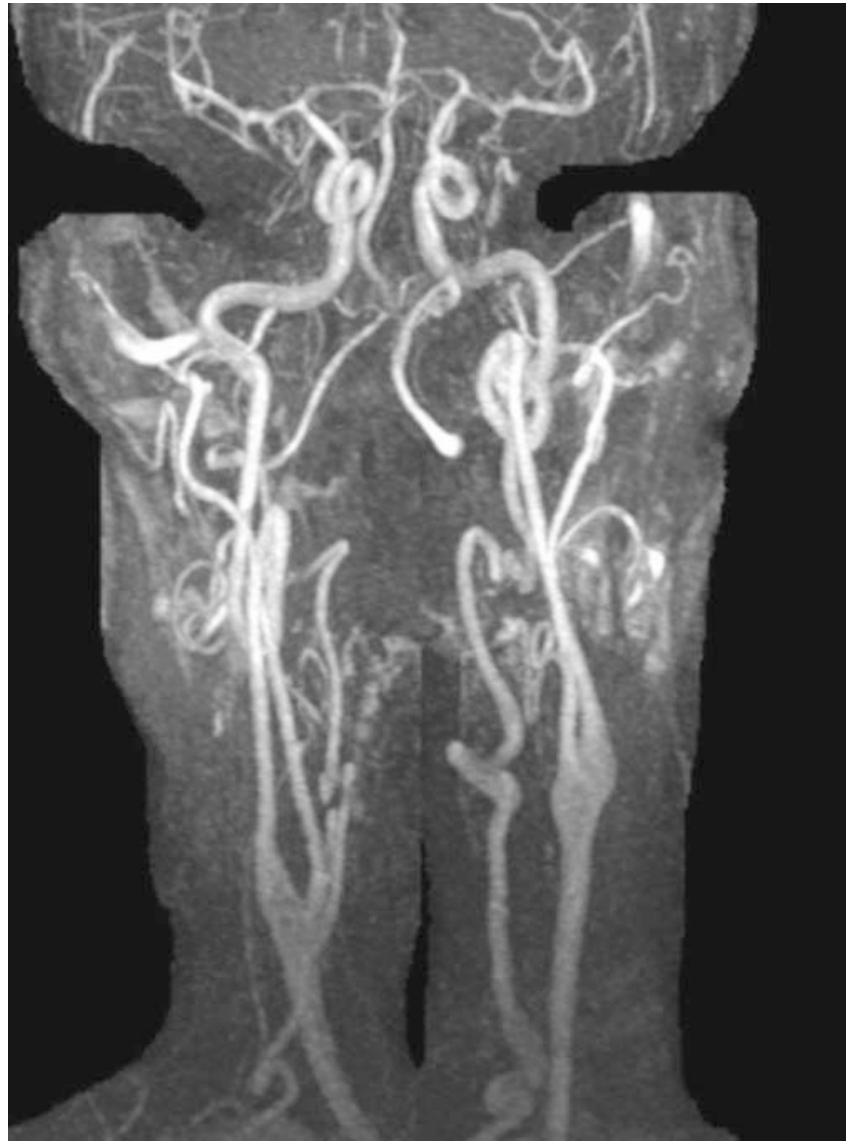

- Fig. 6 Non-contrast time-of-flight (TOF) MRA of the cervical arteries and basal cerebral arteries in 35-year-old Loeys-Dietz patient. Both sides show significant, corkscrew-like tortuosity of the cervical arteries. The tortuosity is not associated with an increased risk of aneurysm or dissection of the affected vessels. However, they are associated with an overall worse prognosis regarding complications of the aorta.

median life expectancy of $40-50$ years. The vascular subtype (IV) based on mutations in the type 3 procollagen gene (COL3A1) occurs in $5 \%$ of cases and has the worst prognosis [46]. Pregnancies in these patients ( $\vee$ Fig. 7A, B) result in a mortality rate of $12 \%$ as a result of uterine rupture or perinatal vascular ruptures [47]. Dissections are rarer in other subtypes. In particular, the kyphoscoliotic type (type $\mathrm{VI}$ ) is associated with the formation of arterial aneurysms and ruptures [48].

Vascular complications of subtype IV affect arterial vessels in up to $82 \%$ of cases. They can occur in various aortic segments as well as in smaller vessels [47]. In contrast to Marfan syndrome, aneurysms of the aortic root are rare and vessel tortuosity as in Loeys-Dietz syndrome is not typical. Elective prophylactic interventions require careful consideration since the fragility of the vessels can result in intraoperative complications like bleeding and dissections.

Experts recommend primary TTE and in the case of an unremarkable finding no further follow-up imaging for adults. Children should be followed-up every three years until the age of 18 [47]. In contrast, a newer study recommends initial visualization
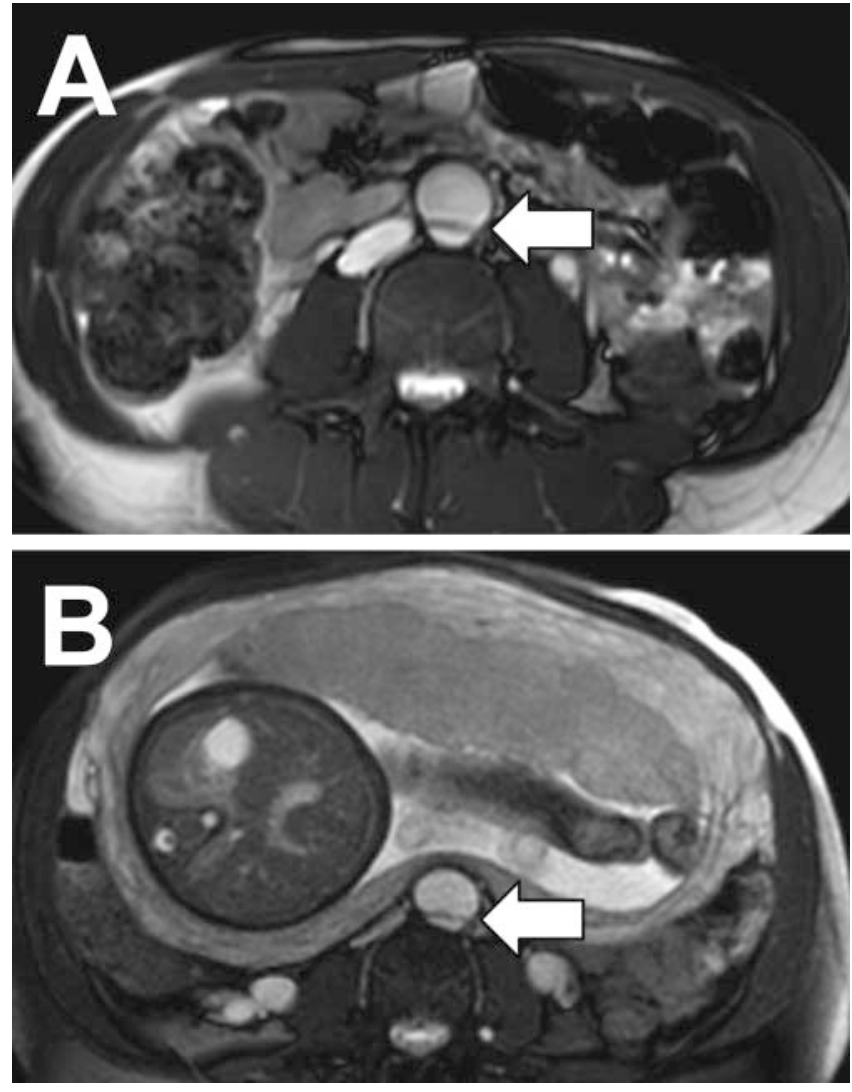

- Fig. 7 Axial non-contrast SSFP-MRA of 29-year-old patient with vascular Ehlers-Danlos syndrome and type B aortic dissection $\mathbf{A}$ before and $\mathbf{B}$ during pregnancy. A The aortic dissection (arrow) was known prior to pregnancy from the screening examinations and extended from the abdominal aorta to the iliac arteries. B The dissection was monitored closely on non-contrast SSFP-MRA during the pregnancy due to the risk of additional complications. The pregnancy progressed without further complications. The dissection also remained limited to the abdominal aorta and the left common iliac artery.

of the entire vascular tree [46]. However, it must be stated that because of the insufficient number of available studies, it is currently not possible to make an evidence-based recommendation regarding the frequency of imaging.

\section{Bicuspid aortic valve disease}

With a prevalence of approx. $1000-2000 / 100000$, bicuspid aortic valve disease is the most common cardiovascular malformation [49]. There is a polygenetic inheritance pattern with variable penetrance frequently associated with mutations of the $\mathrm{NOTCH} 1$ signaling pathway. The inheritance pattern is not yet known [49].

Bicuspid aortic valve disease is a primary disease of the aortic valve but can often result in simultaneous aortic dilatation [50]. With a rate of $0.5 \%$ to $5 \%[51,52]$, aortic dissection is rarer in bicuspid aortic valve disease than in Marfan syndrome [53], but the disease remains a common cause of aortic dissection due to its higher prevalence [54]. 
Dilatation of the ascending aorta can occur in accompanying aortic valve stenosis as well as insufficiency [55]. However, an aneurysm or dissection can form even without stenosis or insufficiency of the bicuspid aortic valve [49, 52]. In addition to a persistent ductus arteriosus, coronary anomalies and brain aneurysms, an aortic isthmus stenosis can also be associated with a bicuspid aortic valve $[49,56]$. This is relevant in that hypertension caused by the aortic isthmus stenosis further increases the risk of dissection.

The Guidelines of the American Association for Thoracic Surgery published in 2018 recommend using TTE for the initial measurement of the proximal aorta [49]. If segments of the aorta cannot be visualized or the aortic diameter exceeds $4.5 \mathrm{~cm}$, ECGtriggered MRA or CTA is recommended. In the case of an aortic isthmus stenosis, the exclusion of intracranial aneurysms is also recommended.

\section{Turner syndrome}

Turner syndrome is characterized by the absence of an X-chromosome and resulting gonadal dysgenesis and dwarfism [57]. It exclusively affects females and has a frequency of approximately $40 / 100000$. Moreover, the cardiovascular mortality rate for these patients is one to three times higher [58]. Apart from aortic isthmus stenosis ( $8 \%)$, bicuspid aortic valve $(10-25 \%)$ is the most common cardiovascular malformation [6].

The majority of dissections in patients with Turner syndrome occur in association with congenital cardiovascular malformations ( $\triangleright$ Fig. 8) [59]. The dwarfism associated with Turner syndrome makes it difficult to assess aortic dilatation: Reference values from a normal-sized population can result in an underestimation in Turner patients. For this reason the aortic diameter determined by Quezada et al. specifically for Turner syndrome should be used [60].

The clinical guidelines of the international Turner Syndrome Symposium from 2016 recommend the following intervals for follow-up imaging depending on age, Z-score, and the presence of three Turner-specific main cardiovascular risk factors: 1. Bicuspid aortic valve, 2. Aortic isthmus stenosis (ISTA), and 3. Arterial hypertension [61]. ISTA is often also associated with further cardiovascular malformations in non-syndromic diseases, such as valve diseases and hypoplasia of the aortic arch [62], so that the term ISTA complex is also used. The range of intervals for follow-up is between 6 and 12 months in the case of a high risk and 5 10 years in the case of a low risk. The recommendation for elective imaging always includes TTE and cardiac MRI including MRA of the entire aorta.

\section{Rarer genetic aortic syndromes}

In addition to the diseases described above, there are a number of further genetic aortic syndromes. These include tortuosity syndrome and aneurysm osteoarthritis syndrome [3]. There are not yet any definitive recommendations regarding imaging for these highly rare diseases.
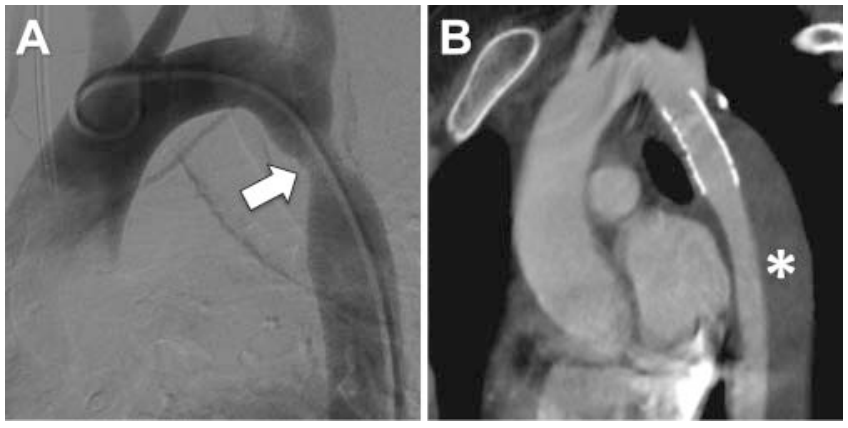

- Fig. 8 32-year-old patient with Turner syndrome $\mathbf{A}$ before and B after treatment of aortic isthmus stenosis. A Periinterventional aortography prior to stent graft treatment of a $40 \%$ aortic isthmus stenosis (arrow). B CT angiography with visualization of a postinterventional type B dissection occurring after stent implantation $\left({ }^{*}\right)$. The patient's aortic isthmus stenosis initially caused treatmentrefractory hypertension that normalized after the intervention.

\section{Summary}

Genetic aortic syndromes are diverse and are highly significant due to their high morbidity and mortality rates. Radiological imaging is essential for diagnosing, following up, and determining the indication for prophylactic aortic root replacement. With knowledge of the special features of genetic aortic syndromes and the recommended imaging techniques, radiology makes an essential contribution to interdisciplinary patient care. Modern imaging technique like 4D flow MRI and pulse wave velocity have the potential to improve individualized risk stratification in patients with genetic aortic syndrome.

\section{Conflict of Interest}

The authors declare that they have no conflict of interest.

\section{References}

[1] von Kodolitsch Y, De Backer J, Schüler $\mathrm{H}$ et al. Perspectives on the revised Ghent criteria for the diagnosis of Marfan syndrome. Appl Clin Genet 2015; 8: 137-155

[2] Judge DP, Dietz HC. Marfan's syndrome. Lancet 2005; 366: 1965-1976

[3] Westerland O, Frigiola A, Robert L et al. Vascular manifestations of syndromic aortopathies: role of current and emerging imaging techniques. Clin Radiol 2015; 70: 1344-1354

[4] Albornoz G, Coady MA, Roberts M et al. Familial Thoracic Aortic Aneurysms and Dissections - Incidence, Modes of Inheritance, and Phenotypic Patterns. Ann Thorac Surg 2006; 82: 1400-1405

[5] Hiratzka LF, Bakris GL, Beckman JA et al. 2010 ACCF/AHA/AATS/ACR/ASA/ SCA/SCAI/SIR/STS/SVM Guidelines for the diagnosis and management of patients with thoracic aortic disease. A Report of the American College of Cardiology Foundation/American Heart Association Task Force on Practice Guidelines, American Association for Thoracic Surgery, American College of Radiology, American Stroke Association, Society of Cardiovascular Anesthesiologists, Society for Cardiovascular Angiography and Interventions, Society of Interventional Radiology, Society of Thoracic Surgeons, and Society for Vascular Medicine. J Am Coll Cardiol 2010; 55 : e27-e129 
[6] Erbel R, Aboyans V, Boileau C et al. 2014 ESC Guidelines on the diagnosis and treatment of aortic diseases: Document covering acute and chronic aortic diseases of the thoracic and abdominal aorta of the adult. The Task Force for the Diagnosis and Treatment of Aortic Diseases of the European Society of Cardiology (ESC). Eur Heart J 2014; 35: 2873-2926

[7] Goldstein SA, Evangelista A, Abbara S et al. Multimodality imaging of diseases of the thoracic aorta in adults: from the American Society of Echocardiography and the European Association of Cardiovascular Imaging: endorsed by the Society of Cardiovascular Computed Tomography and Society for Cardiovascular Magnetic Resonance. J Am Soc Echocardiogr 2015; 28: 119-182

[8] Mortensen K, Baulmann J, Rybczynski M et al. Augmentation Index and the Evolution of Aortic Disease in Marfan-Like Syndromes. Am J Hypertens 2009; 23: $716-724$

[9] von Knobelsdorff-Brenkenhoff F, Gruettner H, Trauzeddel RF et al. Comparison of native high-resolution 3D and contrast-enhanced MR angiography for assessing the thoracic aorta. Eur Heart J Cardiovasc Imaging 2014; 15: $651-658$

[10] Groth M, Henes FO, Müllerleile K et al. Accuracy of thoracic aortic measurements assessed by contrast enhanced and unenhanced magnetic resonance imaging. Eur J Radiol 2012; 81: 762 - 766

[11] François CJ, Hartung MP, Reeder SB et al. MRI for acute chest pain: Current state of the Art. J Magn Reson Imaging 2013; 37: 1290 - 1300

[12] Veldhoen S, Behzadi C, Derlin T et al. Exact monitoring of aortic diameters in Marfan patients without gadolinium contrast: intraindividual comparison of 2D SSFP imaging with 3D CE-MRA and echocardiography. Eur Radiol 2015; 25: $872-882$

[13] Bannas P, Groth M, Rybczynski M et al. Assessment of aortic root dimensions in patients with suspected Marfan syndrome: intraindividual comparison of contrast-enhanced and non-contrast magnetic resonance angiography with echocardiography. Int J Cardiol 2013; 167: 190 - 196

[14] Veldhoen S, Behzadi C, Lenz A et al. Non-contrast MR angiography at 1.5 Tesla for aortic monitoring in Marfan patients after aortic root surgery. J Cardiovasc Magn Reson 2017; 19: 82

[15] Williams JA, Loeys BL, Nwakanma LU et al. Early Surgical Experience With Loeys-Dietz: A New Syndrome of Aggressive Thoracic Aortic Aneurysm Disease. Ann Thorac Surg 2007; 83: S757-S763

[16] Burris NS, Hope MD. 4D flow MRI applications for aortic disease. Magn Reson Imaging Clin N Am 2015; 23: $15-23$

[17] Dyverfeldt P, Bissell M, Barker AJ et al. 4D flow cardiovascular magnetic resonance consensus statement. J Cardiovasc Magn Reson 2015; 17: 72

[18] Markl M, Frydrychowicz A, Kozerke S et al. 4D flow MRI. J Magn Reson Imaging 2012; 36: 1015-1036

[19] Bannas P, Roldán-Alzate A, Johnson KM et al. Longitudinal Monitoring of Hepatic Blood Flow before and after TIPS by Using 4D-Flow MR Imaging. Radiology 2016; 281: $574-582$

[20] Motosugi U, Roldán-Alzate A, Bannas P et al. Four-dimensional Flow MRI as a Marker for Risk Stratification of Gastroesophageal Varices in Patients with Liver Cirrhosis. Radiology 2018: 180230

[21] Chai P, Mohiaddin R. How we perform cardiovascular magnetic resonance flow assessment using phase-contrast velocity mapping. J Cardiovasc Magn Reson 2005; 7: $705-716$

[22] Bannas P, Lenz A, Petersen J et al. Normalization of Transvalvular Flow Patterns After Bicuspid Aortic Valve Repair: Insights From Four-Dimensional Flow Cardiovascular Magnetic Resonance Imaging. Ann Thorac Surg 2018; 106: e319-e320

[23] Potters WV, van Ooij P, Marquering $\mathrm{H}$ et al. Volumetric arterial wall shear stress calculation based on cine phase contrast MRI. J Magn Reson Imaging 2015; 41: 505-516

[24] Wang HH, Chiu HH, Tseng WYI et al. Does altered aortic flow in marfan syndrome relate to aortic root dilatation? J Magn Reson Imaging 2016; 44: $500-508$
[25] Mahadevia R, Barker AJ, Schnell S et al. Bicuspid aortic cusp fusion morphology alters aortic three-dimensional outflow patterns, wall shear stress, and expression of aortopathy. Circulation 2014; 129: 673-682

[26] Geiger ], Hirtler D, Gottfried K et al. Longitudinal Evaluation of Aortic Hemodynamics in Marfan Syndrome: New Insights from a 4D Flow Cardiovascular Magnetic Resonance Multi-Year Follow-Up Study. J Cardiovasc Magn Reson 2017; 19: 1 - 11

[27] Nollen G], Groenink M, Tijssen JGP et al. Aortic stiffness and diameter predict progressive aortic dilatation in patients with Marfan syndrome. Eur Heart J 2004; 25: 1146 - 1152

[28] Whitlock MC, Hundley WG. Noninvasive Imaging of Flow and Vascular Function in Disease of the Aorta. JACC Cardiovasc Imaging 2015; 8: $1094-1106$

[29] Groenink M, de Roos A, Mulder B] et al. Changes in aortic distensibility and pulse wave velocity assessed with magnetic resonance imaging following beta-blocker therapy in the Marfan syndrome. Am J Cardiol 1998; 82: $203-208$

[30] Kröner ES], Scholte AJHA, de Koning PJH et al. MRI-assessed regional pulse wave velocity for predicting absence of regional aorta luminal growth in marfan syndrome. Int J Cardiol 2013; 167: 2977-2982

[31] Flachskampf FA. How Exactly Do You Measure That Aorta? Lessons From Multimodality Imaging. JACC Cardiovasc Imaging 2016; 9: 227 - 229

[32] Elefteriades JA, Farkas EA. Thoracic aortic aneurysm clinically pertinent controversies and uncertainties. J Am Coll Cardiol 2010; 55: 841 - 857

[33] Mendoza DD, Kochar M, Devereux RB et al. Impact of image analysis methodology on diagnostic and surgical classification of patients with thoracic aortic aneurysms. The Ann Thorac Surg 2011; 92: 904-912

[34] Elefteriades JA, Rizzo JA, Coady MA. Thoracic aorta. Radiology 1999; 211: $889-889$

[35] Kehl HG, Kiski D, Orth A et al. Importance of Z scores in congenital heart disease. Z Herz- Thorax- Gefäßchir 2014; 28: 332 - 336

[36] Dormand H, Mohiaddin RH. Cardiovascular magnetic resonance in Marfan syndrome. J Cardiovasc Magn Reson 2013; 15: 33

[37] Engelfriet PM. Beyond the root: dilatation of the distal aorta in Marfan's syndrome. Heart 2006; 92: $1238-1243$

[38] von Kodolitsch Y, Rybczynski M, Vogler M et al. The role of the multidis ciplinary health care team in the management of patients with Marfan syndrome. J Multidiscip Healthc 2016; 9: 587-614

[39] Loughborough WW, Minhas KS, Rodrigues JCL et al. Cardiovascular Manifestations and Complications of Loeys-Dietz Syndrome: CT and MR Imaging Findings. Radiographics 2018; 38: 275-286

[40] Loeys BL, Chen J, Neptune ER et al. A syndrome of altered cardiovascular, craniofacial, neurocognitive and skeletal development caused by mutations in TGFBR1 or TGFBR2. Nat Genet 2005; 37: 275-281

[41] MacCarrick G, Black JH, Bowdin S et al. Loeys-Dietz syndrome: a primer for diagnosis and management. Genet Med 2014; 16: 576- 587

[42] Loeys BL, Schwarze U, Holm T et al. Aneurysm syndromes caused by mutations in the TGF-beta receptor. N Engl J Med 2006; 355: 788 - 798

[43] Lavall D, Schäfers HJ, Böhm M et al. Aneurysms of the ascending aorta. Dtsch Arztebl Int 2012; 109: 227 - 233

[44] Germain DP. Ehlers-Danlos syndrome type IV. Orphanet J Rare Dis 2007; 2: $32-39$

[45] Malfait F, Francomano C, Byers P et al. The 2017 international classification of the Ehlers-Danlos syndromes. Am J Med Genet C Semin Med Genet 2017; 175: 8-26

[46] Eagleton M]. Arterial complications of vascular Ehlers-Danlos syndrome. J Vasc Surg 2016; 64: 1869-1880

[47] Malfait F, De Paepe A. The Ehlers-Danlos syndrome. Adv Exp Med Biol 2014; 802: 129-143 
[48] D’hondt S, Van Damme T, Malfait F. Vascular phenotypes in nonvascular subtypes of the Ehlers-Danlos syndrome: a systematic review. Genet Med 2018; 20: 562 - 573

[49] Borger MA, Fedak PWM, Stephens EH et al. The American Association for Thoracic Surgery consensus guidelines on bicuspid aortic valve-related aortopathy: Full online-only version. J Thorac Cardiovasc Surg 2018; 156 : e41-e74

[50] Verma S, Siu SC. Aortic Dilatation in Patients with Bicuspid Aortic Valve. N Engl J Med 2014; 370: 1920-1929

[51] Itagaki S, Chikwe JP, Chiang YP et al. Long-Term Risk for Aortic Complications After Aortic Valve Replacement in Patients With Bicuspid Aortic Valve Versus Marfan Syndrome. J Am Coll Cardiol 2015; 65: 2363 - 2369

[52] Ward C. Clinical significance of the bicuspid aortic valve. Heart 2000; 83: $81-85$

[53] Michelena HI, Khanna AD, Mahoney D et al. Incidence of aortic complications in patients with bicuspid aortic valves. JAMA 2011; 306: 1104 1112

[54] Wojnarski CM, Svensson LG, Roselli EE et al. Aortic Dissection in Patients With Bicuspid Aortic Valve-Associated Aneurysms. Ann Thorac Surg 2015; 100: 1666-1673-discussion 1673-1674
[55] Aydin A, Desai N, Bernhardt AM] et al. Ascending aortic aneurysm and aortic valve dysfunction in bicuspid aortic valve disease. Int J Cardiol 2013; 164: $301-305$

[56] Schievink WI, Raissi SS, Maya MM et al. Screening for intracranial aneurysms in patients with bicuspid aortic valve. Neurology 2010; 74: 1430 1433

[57] Ho VB. Major Vascular Anomalies in Turner Syndrome: Prevalence and Magnetic Resonance Angiographic Features. Circulation 2004; 110: $1694-1700$

[58] Marin A, Weir-McCall JR, Webb DJ et al. Imaging of cardiovascular risk in patients with Turner's syndrome. Clin Radiol 2015; 70: 803 - 814

[59] Lin AE, Silberbach M. Focus on the heart and aorta in Turner syndrome. J Pediatr 2007; 150: 572 - 574

[60] Quezada E, Lapidus ], Shaughnessy R et al. Aortic dimensions in Turner syndrome. Am J Med Genet A 2015; 167: 2527 - 2532

[61] Gravholt CH, Andersen NH, Conway GS et al. Clinical practice guidelines for the care of girls and women with Turner syndrome: proceedings from the 2016 Cincinnati International Turner Syndrome Meeting. Eur J Endocrinol 2017; 177: G1 - G70

[62] Dijkema EJ, Leiner T, Grotenhuis HB. Diagnosis, imaging and clinical management of aortic coarctation. Heart 2017; 103: 1148-1155 